\title{
A link state aware geographic routing protocol for vehicular ad hoc networks
}

\author{
Changle Li ${ }^{*}$, Liran Wang, Ying He, Chunchun Zhao, Hang Lin and Lina Zhu
}

\begin{abstract}
In vehicular ad hoc networks (VANETs), the communication links are inherently unstable due to vehicles' mobility and various impairment of radio signal. Existing geographic routing protocols often choose the next hop according to the greedy forwarding, regardless of the link's quality and transmission reliability. The successful packet delivery rate is decreased in non-ideal communication links. Consequently, the reliability of data transmission is worse and the network throughput is declined. In this paper, we propose a routing protocol called link state aware geographic routing protocol (LSGR) for VANETs. In LSGR, a routing metric called expected one-transmission advance (EOA) is contrived to improve the greedy forwarding algorithm by explicitly incorporating the link state and packet's advance. Routing with the EOA metric, one can improve the transmission efficiency by diminishing transmission failures. Simulation results show that LSGR can achieve a higher throughput and packet delivery rate than the geographic routing protocols that adopt the traditional greedy forwarding.
\end{abstract}

Keywords: VANETs; Geographic routing; Greedy forwarding; EOA

\section{Introduction}

Vehicular ad hoc networks (VANETs) open up the opportunity to develop powerful traffic systems capable of gathering, processing, and distributing information by vehicle-to-vehicle (V2V) and vehicle-to-roadside (V2R) communications. VANETs have attracted wide interest of the research groups because of the great potential to improve the traffic safety, efficiency, and convenience [1]. For example, nearby vehicles can inform each other about their positions and velocities by broadcasting short messages to avoid collisions and mortality in thick fog. A driver can select a path which is short and without congestion through the traffic information released by the infrastructures. Therefore, VANETs possess great social values and commercial benefits.

With the increasing demand for these applications to connect to the Internet, the IP mobility support of vehicular communication reveals its importance. The Network Mobility Basic Support (NEMO BS) [2] is an important protocol to ensure IP mobility for the reachability of mobile nodes to the Internet. The NEMO BS is intended to provide continuous connection for a group of nodes on

\footnotetext{
*Correspondence: clli@mail.xidian.edu.cn

State Key Laboratory of Integrated Services Networks, Xidian University, Xi'an,
} Shaanxi 710071, China move. A mobile router (MR) is utilized to manage the connection of a group of nodes. It is a potential candidate for providing IP mobility in VANETs. Meanwhile, there are limitations for NEMO BS to apply to VANETs at the same time. Firstly, it does not provide multi-hop connections to the infrastructure. Baldessari et al. [3] proposed two approaches to integrate MANET routing protocol with NEMO, thus came to a VANEMO solution. Secondly, it cannot adapt to high dynamic scenarios; Céspedes et al. [4] compared several IP mobility solutions for optimizing NEMO BS to better perform in vehicular scenarios, incorporating the route optimization (RO). Thirdly, according to NEMO BS, when a vehicle moves around, it needs to register a new IP address for new access of the network, resulting in long handover latency and high traffic load. Lee et al. $[5,6]$ provided a mechanism utilizing the mobility service provisioning entities in PMIPv6 so that vehicles can keep their connectivity to the Internet without updating their location information. Analysis and simulation have been done to compare the performance of proposed protocol and NEMO BS, and the improvement in handover performance will be a positive force in the research of IP mobility solutions.

Another foundation to guarantee the performance of these applications is the efficient data transmission. Thus

\section{照 Springer}

C $2014 \mathrm{Li}$ et al: licensee Springer. This is an Open Access article distributed under the terms of the Creative Commons Attribution License (http://creativecommons.org/licenses/by/2.0), which permits unrestricted use, distribution, and reproduction in any medium, provided the original work is properly credited. 
routing strategy is an essential part that influences the performance of the network. Recently, many works have provided in-depth studies of the link quality of VANETs [7-9]. Based on these researches, we can reach a conclusion that because of the dynamic nature of VANETs and wireless channel fading, individual links present unstable connectivity. The link quality has a relationship with the distance between the corresponding nodes, and the nearer the nodes are, the better the link quality they have.

Confront with the challenges in VANETs, geographic routing [10-18] is commonly regarded as highly scalable and a very robust protocol against frequent changes. Such routing strategies route packets according to the position of the involved nodes, i.e., the forwarder, the neighbors of the forwarder, and the destination. Greedy forwarding is the most widely adopted strategy in geographic routing. This strategy chooses the neighbor that has the shortest distance to the destination as the next hop so that each hop makes the greatest progress dropping ratio towards the destination. However, such a strategy will probably minimize signal strength and maximize the packet dropping ratio [19]. This is due to the fact that the next hop obtained in this way is largely located close to the edge of the transmission range of the forwarder. As the packet is forwarded using links with a high dropping ratio, if the forwarding node MAC protocol uses the retransmission mechanism, an excessive amount of network bandwidth and time will be consumed by retransmissions. As a result, the network throughput is declined and the end-to-end delay is prolonged. Studies were made of the disadvantages of using the greedy forwarding based on the link quality $[14,15,20,21]$. The evaluation criterion of the link quality is usually the energy power of the received signal [20], and the link quality is related strongly to the distance between the forwarding node and the intermediate node [21].

Different from the researches above, De Couto et al. proposed the expected transmission count (ETX) metric [22] to evaluate the quality of a certain link. The ETX of a link demonstrates the expected number of transmissions required for sending a packet over the link, and a better quality link has a smaller value of ETX. It aims at finding high-throughput paths which minimize the expected total number of MAC-layer transmissions (including retransmissions) required for delivering a packet hop-by-hop to its destination. The ETX routing metric has been shown effective in selecting good quality routes [22,23] and is widely used in routing protocols for wireless multi-hop networks [24-26]. However, ETX is used mainly in opportunistic and proactive routing protocols which are developed for low-speed mobile ad hoc networks (MANETs). The research about adopting ETX routing metric in geographic routing for VANETs is still in its infancy. The difficulty in using ETX in VANETs is that ETX does not specifically account for mobility. Consequently, ETX must be modified to adapt to the highly dynamic network environment.

Intuitively, the link between two nodes which are close to each other has a high delivery rate. Accordingly, its ETX will be close to 1 . However, such link cannot make much contribution to the packets advance on the destination. Therefore, a tradeoff should be made between the packet advance and transmission reliability. For this reason, we propose a routing metric called expected one-transmission advance (EOA) to improve the greedy forwarding. The EOA indicates the average geographic distance that a packet can make through one transmission. Instead of choosing the neighbor that is geographically closest to the destination, the neighbor with the largest EOA will be chosen as the next hop.

In this paper, we improve the greedy forwarding strategy by taking the link state into account. The link state is measured by the ETX metric which is obtained with the assistance of the Hello scheme making every node in the network broadcast short packets periodically to inform neighbors of its own information (e.g., position and velocity). The calculation of ETX is modified to adapt to the high mobility of the network nodes. Based on the ETX metric, we propose EOA metric to decide the next hop. Our proposed routing protocol is considered as link state aware; it can be reflected by the fact that each node maintains the expected transmission counts for a packet to be successfully transmitted to its neighbors. Along with the movement of the vehicles, the expected transmission counts needed are updated periodically. Thus the link state is measured and utilized as the basis for the choice of the next hop. The contribution of this paper can be summarized as the following points.

1. We amend the method to calculate the ETX of a specific link. The calculation explicitly takes the mobility of nodes into account and is well adapted to the dynamic networks.

2. A routing metric called EOA is produced to improve the greedy forwarding strategy. The enhanced greedy forwarding algorithm forwards the packets by incorporating the link state and the geographic distance. It can largely diminish transmission failures.

3. We propose a routing protocol called link state aware geographic routing protocol (LSGR) for VANETs. LSGR adopts the enhanced greedy forwarding algorithm. It has the advantages of increasing the network throughput and reducing the transmission delay.

The rest of the paper is organized as follows. Section 2 reviews some existing routing protocols designed for VANETs. Section 3 describes the optimized forwarding 
mechanism and details other components of the protocol. Section 4 presents the results of the performance evaluation of the proposed protocol and makes an analysis of the results. Finally, Section 5 concludes the paper.

\section{Related work}

The routing protocols in VANETs can be generally classified into two categories, topology-based and positionbased $[27,28]$. Topology-based protocols use the available link state information to perform packet forwarding $[29,30]$. It is assumed that each node has information about the entire network topology before a packet is being sent out. Such strategies will generate a large amount of network overhead in VANETs. The prerequisite of position-based routing is the knowledge of the position of the forwarder, its neighbors, and the destination. The increasing availability of GPS-equipped vehicles and location service [31] provides a guarantee. Geographic routing protocols have the advantages of good scalability with respect to the network size and adaptability to the highly dynamic network.

In geographic routing protocols, greedy routing protocols are considered most suitable for the unique characteristics of VANETs. The greedy algorithm is applied to solve the routing problem and has been well defined in [32]. The source node forwards a message to the neighboring node that is closest to the destination. The information needed to route a packet is only the physical positions of its neighbors and the destination, and these positions can be obtained by the periodically broadcast beacons. Greedy routing does not require the establishment or maintenance of routes, and can be well adapted to the high dynamic scenarios of VANETs.

Greedy perimeter stateless routing (GPSR) [10] is a wellknown geographic routing protocol in wireless multi-hop networks. The routing algorithm consists of two parts, a greedy forwarding mode and a perimeter mode. Greedy forwarding is the default mode, and the packets are initially routed in this mode. When there is a void between the forwarding node and destination node (called a local optimum), namely the forwarding node cannot find a neighbor which is geographically closer to the destination than itself, GPSR switches into the perimeter mode. A distributed algorithm is first implemented to obtain a planar graph. Then the packets are routed along the perimeter of the void based on the right-hand rule and look for an opportunity to return to the greedy mode (Figure 1). However, routing with greedy forwarding in GPSR may lead to wrong directions, and there are too many hops for the packet to be transmitted to the destination. Greedy perimeter coordinator routing (GPCR) [11] improves GPSR to better adapt to the VANETs in city scenarios by separating the routing on streets from junctions. In every street, a special form of greedy forwarding

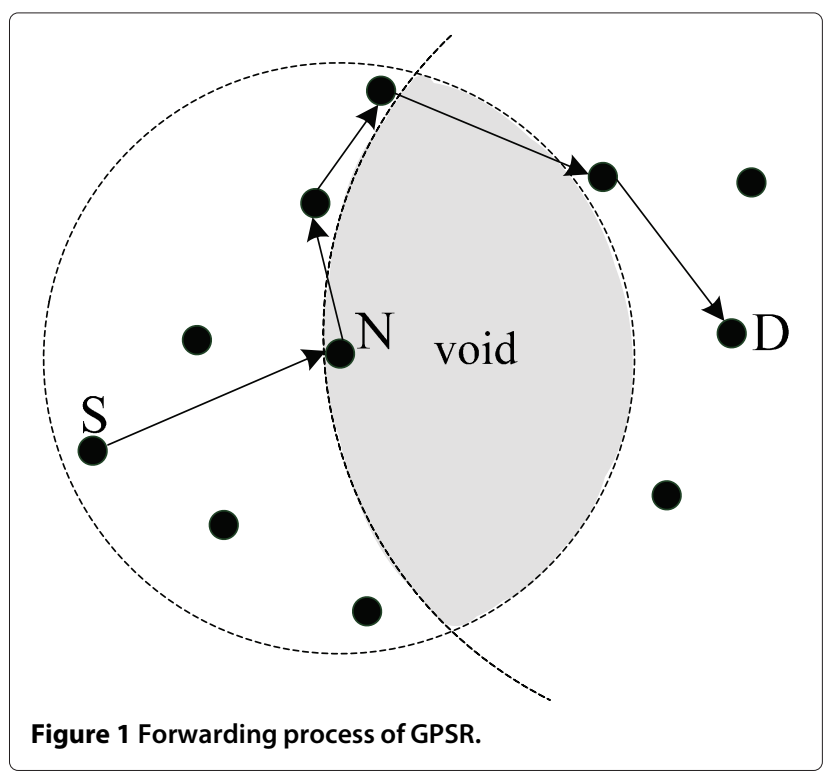

is used to forward the packet towards the next junction. On each junction, the forwarder has to decide which street the packet should be followed next. The repair strategy of GPCR avoids using graph planarization by taking advantage of the natural planar graph formed by the streets and junctions. In GPCR, end-to-end connections are difficult to establish in low traffic density.

VADD [33] is designed for the frequently disconnected networks. It implements the carry and forward scheme. When a node fails to find a neighboring node to forward the packet to, it stores the packet until a new node arrives to its transmission range, hence the end-to-end delay is large in sparse networks. Greedy forwarding is utilized to forward packets along the streets. A delay model is proposed to calculate the packet delivery delay of each candidate street, and the street with the lowest delay is selected to forward the packet.

Since the packets are generally forwarded based on the greedy forwarding along the streets, existing geographic routing protocols mostly focus on the routing on junctions, i.e., which street is followed. GyTAR [12] sequentially chooses the intersections considering the remaining distance to the destination and the variation in vehicular traffic. An improved greedy strategy that predicts the position of each neighbor before choosing the next hop is utilized to forward data between two intersections. GyTAR is good at finding a robust routing in city environments; however, GyTAR does not consider the directions of the vehicles in the junction selection mechanism. When there are vehicles on the opposite road of the desired destination, the protocol suffers a large end-to-end delay and low packet delivery rate. STAR [13] explores the impact of traffic lights on a routing protocol. Since vehicles tend to cluster in front of the two sides of the road segments 
with red lights, the choice of the next road is based on the policy of green light roads first. In HTAR [14], a forwarding node at a junction adaptively decides on a routing path according to the real-time hybrid traffic information, which includes the node density, distance, and network traffic load of the candidate roads.

The geographic routing protocols mentioned above always forward the packets to each intermediate intersection to make the routing decision. Lee et al. [15] noted the fact that packets do not necessarily need to stop at each junction if the transmission direction does not change and proposed GpsrJ+. The segment to which the packet will be forwarded is pre-computed. If the transmission direction does not change, the forwarder simply bypasses the junction node and forwards the packet to its furthest neighbor; otherwise, it will forward to the junction node. However, GpsrJ+ is not suitable for delaysensitive applications or actual city maps, since it assumes the road as a simple straight line while the actual city map has more complicated roads. Afterwards, Lee et al. proposed another protocol called TO-GO [16], which incorporates geographic routing with opportunistic forwarding. TO-GO utilizes the two-hop information to make an advanced decision on whether to bypass the junction.

All of these routing protocols have proposed various strategies to improve the performance of geographic routing in VANETs. However, few of them have made an improvement to the greedy forwarding. In this paper, we will propose an enhanced greedy forwarding strategy to improve the performance of geographic routing protocols in VANETs.

\section{Link state aware geographic routing protocol}

In this section, we introduce our proposed routing protocol LSGR. Firstly, we describe the method utilized to measure the link state in VANETs. Then, the EOA metric is introduced, and finally the LSGR algorithm is presented in detail.

\subsection{Computing ETX in VANETs}

First, we briefly introduce the calculation method presented in [22]. In IEEE 802.11, a packet is considered to be successfully transmitted after the sender receives the acknowledgment packet from the receiver. Therefore, the calculation of ETX takes the asymmetric loss rates into account. The case of the link between the nodes $\mathrm{X}$ and $\mathrm{Y}$ is shown in Figure 2. Suppose $d_{f}$ is the forward packet delivery probability from $\mathrm{X}$ to $\mathrm{Y}, d_{r}$ is the reverse packet delivery probability from $\mathrm{Y}$ to $\mathrm{X}$. The ETX of the link is obtained by

$$
\operatorname{ETX}=\frac{1}{d_{\mathrm{f}} \cdot d_{\mathrm{r}}} .
$$

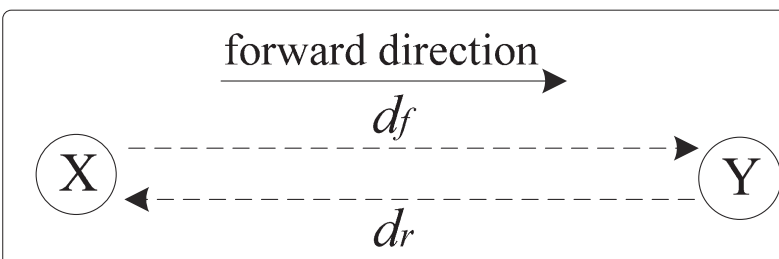

Figure 2 The link between $X$ and $Y$.

The delivery probability $d_{f}$ and $d_{r}$ are measured using dedicated link probe packets, which are broadcasted at an average period $\tau$. A window lasting $w$ seconds is used to measure the delivery probability. Every node remembers the number of probes it has received during the last $w$ seconds, allowing it to calculate the delivery probability from the sender at any time $t$ as

$$
r(t)=\frac{\operatorname{count}(t-w, t)}{w / \tau}
$$

count $(t-w, t)$ is the number of probes that the node has received at time $t$ during the window $w$, and $w / \tau$ is the number of probes that should have been received. For a link shown in Figure 2, node X can only calculate $d_{r}$ and $\mathrm{Y}$ can only calculate $d_{f}$. In order to allow every node to measure the ETX of a link, each probe sent by a node contains the number of probe packets it has received from each of its neighbors during the last $w$ seconds.

As can be seen from the description, ETX does not specifically account for mobility. In the calculation of ETX, $w / \tau$ is the number of probes that should have been received during the window $w$; however, in highly mobile networks, the probability should be calculated after the node enters into the transmission range of its neighboring node. Suppose that node $X$ enters into the transmission range of node $\mathrm{Y}$ at time $t_{0}$. Then $\mathrm{Y}$ needs to measure the packet delivery rate from $\mathrm{X}$ to $\mathrm{Y}$ at time $t\left(t-t_{0}<w\right)$. The result obtained by Equation 2 is wrong because of the inappropriate probe count that should be received. Assume that the broadcast interval of the probes is $1 \mathrm{~s}$, the length of the window $w$ is $10 \mathrm{~s}$, and $t-t_{0}=6 \mathrm{~s}$. During this time, the total number of probes that $\mathrm{Y}$ received from $\mathrm{X}$ is 5 . The packet delivery rate from $\mathrm{X}$ to $\mathrm{Y}$ is $5 / 6=83.33 \%$. However, the result from Equation 2 is $5 / 10=50 \%$.

Therefore, we adjust the calculation of ETX to adapt to the networks that are highly dynamic. Note that Hello packets are periodically broadcasted to record the information of neighbors in several geographic routing protocols. We use the Hello packets instead of dedicate probe packets to measure the packet delivery probability in LSGR. Traditionally, each node maintains a neighbor table that records the position information of every neighbor. To calculate the ETX of a link, the neighbor table should 
record the time $\left(t_{0}\right)$ when the first Hello packet is received from a neighbor and the number of Hello packets it has received from the neighbor during the last $w$ seconds. The entry of a node in the neighbor table is shown in Figure 3. According to the window $w$ and the interval between $t_{0}$ and the current time $t$, the calculation formula is

$$
r(t)= \begin{cases}\frac{\operatorname{count}(t-w, t)}{w / \tau}, & t-t_{0} \geq w \\ \operatorname{count}\left(t-t_{0}\right), & 0<t-t_{0}<1 \\ \frac{\operatorname{count}(t-w, t)}{\left(t-t_{0}\right) / \tau}, & 1 \leq t-t_{0}<w\end{cases}
$$

As can be seen from the formula, there are three situations in terms of the difference between window $w$ and $t-t_{0}$. 1) $t-t_{0} \geq w$, in this situation, the calculation is the same as Equation 2. 2) $0<t-t_{0}<1$, the time difference between the current time and $t_{0}$ is less than $1 \mathrm{~s}$. In this case, the packet delivery rate is the number of Hello packets received from $t_{0}$ to $t$. Note that the probability is certainly equal to 1 . If count $\left(t_{0}, t\right)$ is divided by $t-t_{0}$, the result would be a very large number. 3) $1 \leq t-t_{0}<w$, the packet delivery probability in this condition is the number of the Hello packets received from $t_{0}$ to $t$ divided by the number of Hello packets should have been received during this period.

Note that in the Hello scheme, the entry of a neighbor will be deleted from the neighbor table if the node has not received a Hello packet from the neighbor for a period of time. We set this time to be twice the broadcast interval. Then, the minimum packet delivery probability of a link is $1 / 3=33.33 \%$. Accordingly, the maximum ETX of a link would be $1 /(0.33 \times 0.33)=9.18$. The distribution of ETX value of the link is correlated with the parameters that are set in the Hello scheme.

\subsection{Routing based on link state}

In LSGR, the next hop is selected using an enhanced greedy forwarding, which performs according to the EOA routing metric. The EOA value indicates the expected advance that a packet can make towards the destination through one transmission. Instead of choosing the neighbor that is geographically closest to the destination, the enhanced greedy forwarding chooses the neighbor with the largest EOA. When a node $S$ has a packet to forward, it calculates the geographic advance each neighbor can make and the expected number of data transmissions require to successfully send a packet over the link formed by $\mathrm{S}$ and the neighbor. Then, the EOA of neighbor $i$ is obtained by

$$
\mathrm{EOA}_{i}=\frac{D_{\mathrm{ns}}-D_{\mathrm{ni}}}{\mathrm{ETX}_{i}}
$$

where $D_{\mathrm{ns}}$ is the distance from the forwarding node to the destination node. $D_{\text {ni }}$ is the distance between the neighbor $i$ and the destination node. ETX $\mathrm{ET}_{i}$ is the ETX of the link that is formed by the forwarding node and the neighbor $i$. $D_{\mathrm{ns}}-D_{\mathrm{ni}}$ indicates the geographic distance a packet can advance towards the destination. However, due to the link loss, to be successfully forwarded over that link, a packet needs to be transmitted ETX $_{i}$ times on average. Hence, a neighbor's EOA demonstrates the expected advance that a packet can make towards the destination through one transmission if it chooses the neighbor as the next hop.

EOA metric incorporates the geographic distance and the link quality. It is a tradeoff between the advance and transmission reliability. It tends to minimize the bandwidth use and increase the network throughput by taking the link state into account. With less transmission times, EOA can also reduce the end-to-end delay.

\subsection{Routing on junctions}

Routing in VANETs, especially in city scenarios, usually separates the streets from junctions. In LSGR, the enhanced greedy forwarding is adapted to route packet in streets. On junctions, LSGR incorporates the distance and the network connectivity to choose the next street. The network connectivity of a street is reflected by the vehicle density in the street. A distributed algorithm has been proposed in our previous work [18] to collect the vehicle density of a certain street. In LSGR, we use the algorithm to get the vehicle density of the streets. With the algorithm, the following parameters of a street can be obtained:

$N_{\text {avg }}$ is the average number of vehicles of a unit scope. The unit scope is defined as a road segment whose length is equal to the transmission range of a node, as shown in Figure 4, in which the value of $N_{\text {avg }}$ is $11 / 3=3.67$.

$N_{\text {min }}$ is the minimum number of vehicles among the unit scopes in a certain street. In the street shown in Figure 4, the value of $N_{\min }$ is 2 .

These parameters are obtained with the help of Network Information Collection Packet (NICP) in three steps: 1. When a vehicle is at the junction, a NICP is generated with the number of its neighbors as $N_{\text {sum }}$ and $N_{\min }$. 2. The

\begin{tabular}{|l|l|c|c|c|}
\hline Node ID & Time stamp & $\begin{array}{c}\text { Time when first receives } \\
\text { a hello packet from this } \\
\text { neighbor }(t o)\end{array}$ & $\begin{array}{c}\text { Number of hello packets } \\
\text { received during the last } \\
w \text { seconds }\end{array}$ & position \\
\hline
\end{tabular}

Figure 3 The format of an entry in the neighbor table. 


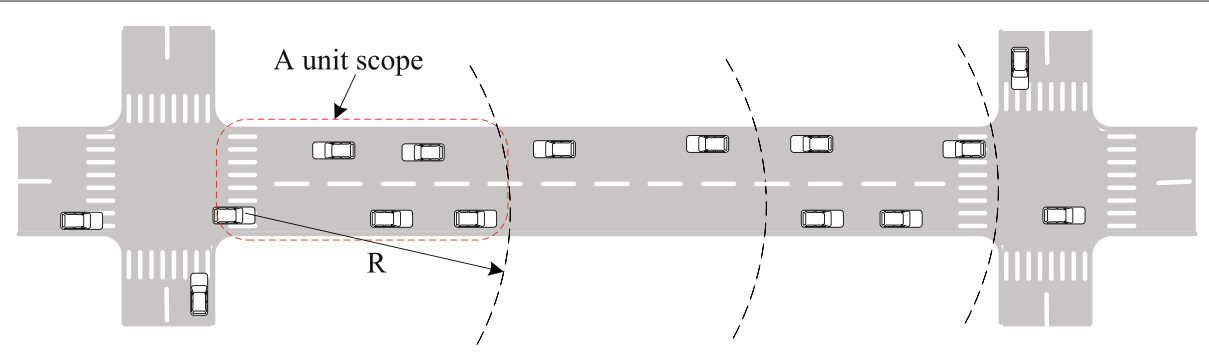

Figure 4 Vehicle density in a street.

NICP is forwarded to its farthest neighbor as a receiver, and $N_{\text {sum }}$ is modified by adding the number of neighbors on the forwarding side (in Figure 4, the right side) of the receiver, $N_{\min }$ is modified to the number of the receiver's neighbors in the forwarding side if it is less than $N_{\min } .3$. Repeat this process until the NICP is forwarded to another junction, then $N_{\text {sum }}$ and $N_{\text {min }}$ of this road segment can be obtained.

A node at junction chooses the next street to transmit the packet according to the following function

$$
S(J)=\alpha \times(1-D)+\beta \times \frac{N_{\mathrm{avg}}}{1+\left(N_{\mathrm{avg}}-N_{\min }\right)},
$$

where $D=D_{\mathrm{j}} / D_{\mathrm{i}}, D_{\mathrm{j}}$ is the distance from the midpoint of the candidate street to the destination and $D_{\mathrm{i}}$ is the distance between the current junction and the destination. $\alpha$ and $\beta$ are weighting factors. $S(J)$ is the score of other adjacent road segments that integrates the distance and the network connectivity, and the road segment with highest $S(J)$ will be chosen to forward the packet to. $D$ is the closeness of the candidate junction to the destination, the shorter the distance from the candidate junction to the destination, the lager the value of the first item. $N_{\text {avg }}$ is the average number of vehicles in a unit scope, $N_{\text {avg }}-N_{\text {min }}$ reflects the uniformity degree of the distribution of the vehicles in the road segment, and the road segment with large and balanced vehicle density has more opportunity to be chosen.

Now we will have a brief discussion on the influence of the vehicular traffic in three conditions with respect to different values of $N_{\min }$ :

1. When $N_{\min }=0$, that means on this road segment there is a vehicle having no neighbors in the forwarding direction of itself, thus the network on this road segment is considered disconnected. At this time the NICP cannot be delivered from one junction towards the other side; therefore, this road segment will not be selected until after a period of time $N_{\min }$ grows larger than 0 due to the movement of the vehicles.
2. When $1 \leq N_{\min }<N_{\text {avg }}$, at this time, the uneven node distribution is penalized by the denominator, making the second item a smaller value. The closer these two values are, the smaller the penalization it has.

3. When $N_{\min }=N_{\text {avg }}$, then value of the second item without weighting factor equals to $N_{\text {avg }}$, the road segment is connected and the distribution of vehicles on this road segment is approximately uniform, and it can be reflected by the relatively higher value of the second item. Then the road segment with such characteristic has higher priority to be chosen to forward the packet to.

\subsection{Repair strategy}

When the network nodes become sparse, there is a high probability that a packet gets stuck in a local optimum. In this case, the forward algorithm enters into a repair strategy. In LSGR, the repair strategy combines the idea of carry and forward [17] and the perimeter forwarding. The current vehicle in a repair mode will carry the packet for a period of time to look for an available neighbor to forward the packet. If the vehicle cannot find an available next hop during the period, the packet is forwarded back to the last junction. Then the packet is forwarded back in the perimeter mode similar to that in GPCR. In the simulation in Section 4, the period is dynamically set as $R / 2 v$. Where $R$ is the transmission range and $v$ is the speed of the vehicle when it begins to carry the packet.

\subsection{Further discussion}

EOA incorporates the distance and the link quality to choose the next hop. It has the advantages of increasing the network throughput and reducing the end-to-end delay. However, several deficiencies of EOA should be illuminated here. And our future work will focus on perfecting the routing metric.

1. The ETX of a link is measured by the link's packet delivery probability, which is obtained through the Hello packets. Note that the Hello packet is a short 


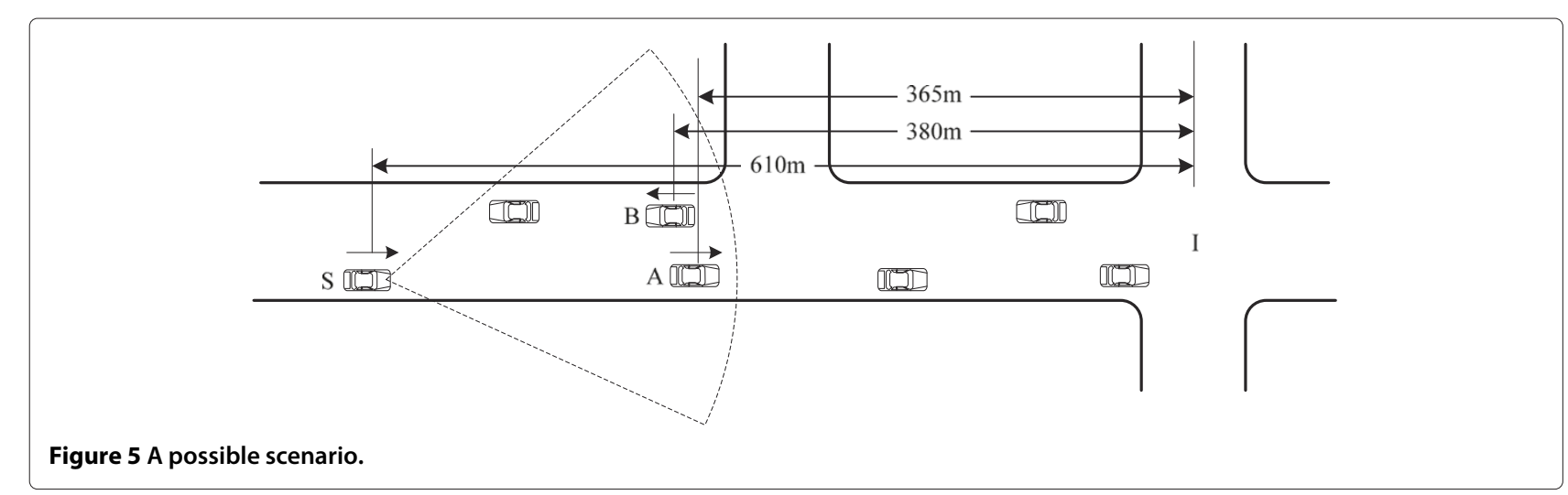

packet which can be much shorter than a data packet. In wireless networks, a long packet is more vulnerable to bit error and packet dropping than a short packet. The chance of a Hello packet dropping is comparatively much smaller than that of a data packet dropping. Therefore, while glaring discrepancies exist among the ETX of different links for data packets, it is not for the Hello packets. This fact will mislead the nodes about the choice of the next hop, making distance become the dominant factor.

2. Suppose such a scenario, where vehicles $A$ and $B$ are two neighbors that are located close to the transmission range of vehicle $S$, as shown in Figure 5. A travels in the same direction as $\mathrm{S}$, and the connectivity between them have existed for a period of time. Whereas B travels in the opposite direction and it has just entered the transmission range of $\mathrm{S}$.
After B enters the transmission range of $\mathrm{S}$, it broadcasts a Hello packet and it is successfully received by $S$. In less than $1 \mathrm{~s}$ after $S$ receives the Hello packet from B, S needs to forward a packet which is destined for the intersection I. Assume that ETX of the link between $S$ and $A$ is 1.65. As the time when $S$ received the first Hello packet from $B$ is less than $1 \mathrm{~s}$ before the current time, ETX of the link between $\mathrm{S}$ and $\mathrm{A}$ computed by $\mathrm{S}$ is 1 . As a sequence, the EOA of A and B are 148.48 and 230, respectively. Then vehicle $B$ will be chosen as the next hop. However, obviously the calculation result from one Hello packet cannot fully reflect the state of the link between $\mathrm{S}$ and B. An alternative method to address this problem is to take the moving direction into account. The neighbors which are moving in the same direction as the forwarder takes priority over those that move in the opposite direction.

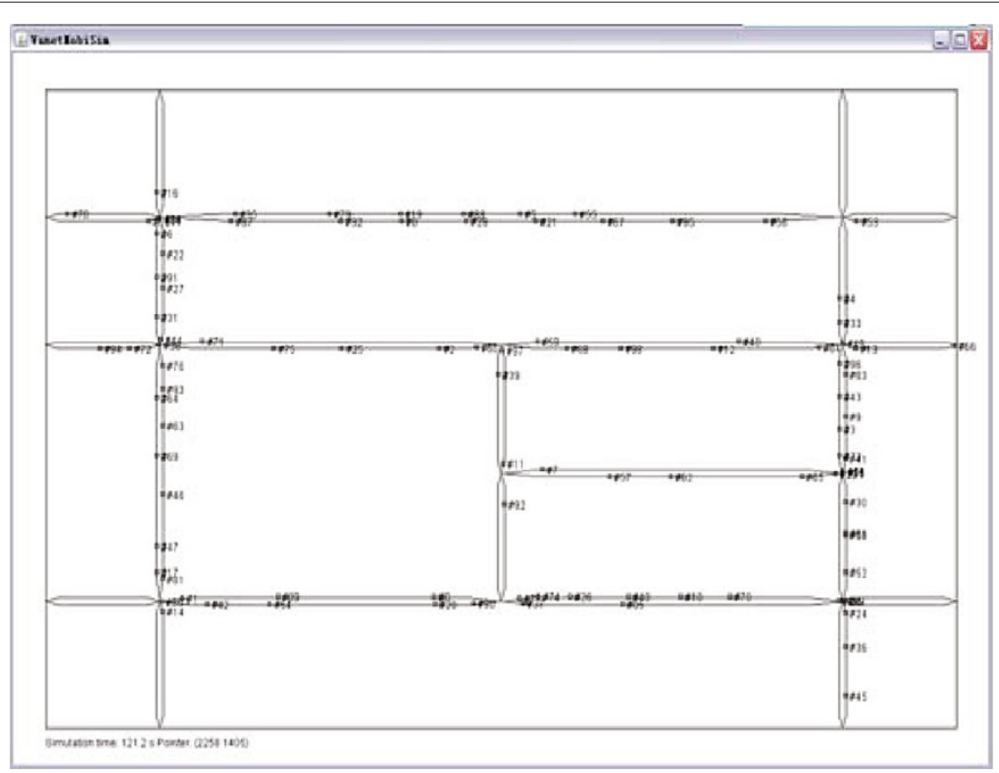

Figure 6 Simulation scenario. 


\section{Performance evaluation}

In this section, we evaluate the performance of LSGR and compare it with GpsrJ+ [15] and GyTAR [12] via the network simulator NS-2 [34].

\subsection{Simulation setup}

We simulate the protocols in a $2,500 \times 1,500 \mathrm{~m}$ rectangle scenario that is formed by gridded streets, as shown in Figure 6 . The map is generated by the vehicular mobility model generator VanetMobiSim [35], an open source program which can generate more realistic vehicular mobility for NS-2. There are 100 to 200 vehicles randomly distributed initially in the junctions. Once the simulation begins, each vehicle moves at a speed ranging from 20 to $80 \mathrm{~km} / \mathrm{h}$ along a path towards a randomly chosen destination. The instantaneous speed of a vehicle depends on nearby cars. Ten pairs of communication nodes are randomly chosen among the vehicles in each simulation. Each source node sends packets at the rate of 1 Mbps with a packet size of 512 bytes. The propagation model used in the simulation is the two-ray ground model and the transmission range of each vehicle is set to $250 \mathrm{~m}$. We set the value of the Hello interval as $1 \mathrm{~s}$ and the window size $w$ as $10 \mathrm{~s}$. Since the ETX metric depends highly on these two parameters, before comparing LSGR with other protocols, we first simulate the impact of the value of the Hello interval and window size $w$. The weighting factors $(\alpha, \beta)$ are set to $(0.5,0.5)$. All the key parameters of our simulation are summarized in Table 1.

We evaluate the performance of the protocols by four metrics:

1) End-to-end delay is defined as the average amount of time spent by the transmission of a packet that is

Table 1 Simulation parameters

\begin{tabular}{ll}
\hline Parameter & Default value \\
\hline Map size & $2,500 \times 1,500 \mathrm{~m}$ \\
Mobility model & VanetMobiSim \\
Number of vehicles & 100 to 200 \\
Vehicles speed & $20 \sim 80 \mathrm{~km} / \mathrm{h}$ \\
MAC protocol & IEEE $802.11 \mathrm{DCF}$ \\
Propagation model & Two-ray ground model \\
Channel rate & 2 Mbps \\
Transmission range & $250 \mathrm{~m}$ \\
Traffic model & $10 \mathrm{CBR}$ connections \\
Packet rate & $0.5 \mathrm{~s} /$ packet \\
Hello interval & $1 \mathrm{~s}$ \\
Data packet size & 512 bytes \\
Weighting factors $(\alpha, \beta)$ & $(0.5,0.5)$ \\
\hline
\end{tabular}

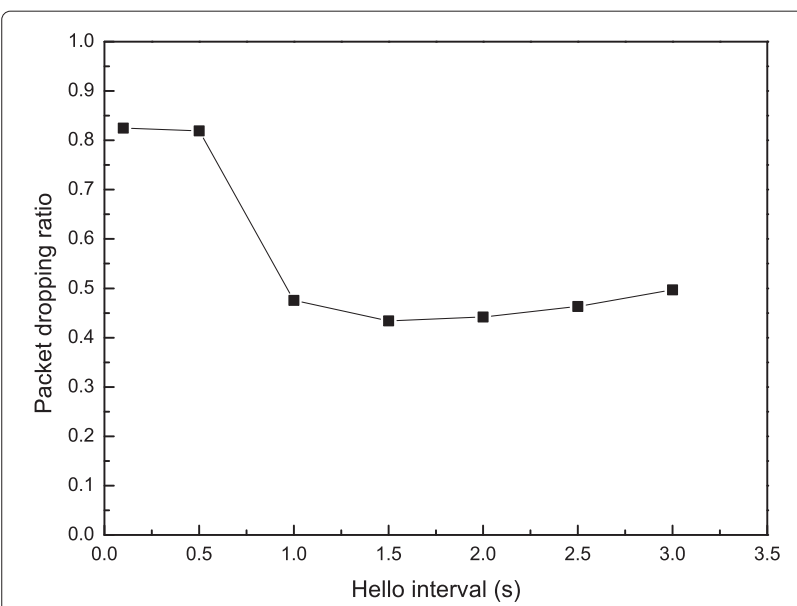

Figure 7 Packet dropping ratio vs. the Hello interval.

successfully delivered from the source to the destination.

2) Hop count is defined as the average number of hops that the packets forwarded from the source to the destination.

3) Packet delivery rate is defined as the ratio of the number of packets successfully delivered to the destination to the number of the total packets generated in the simulation.

4) Network throughput is defined as the number of bits successfully transmitted per second in the network.

\subsection{Simulation results}

\subsubsection{The impact of the Hello interval}

In the simulation, the number of vehicles is set to 120 , and the window size $w$ is $10 \mathrm{~s}$. We vary the value of Hello interval from 0.1 to $3 \mathrm{~s}$ to see the impact of the Hello interval on the packet dropping ratio and the end-to-end

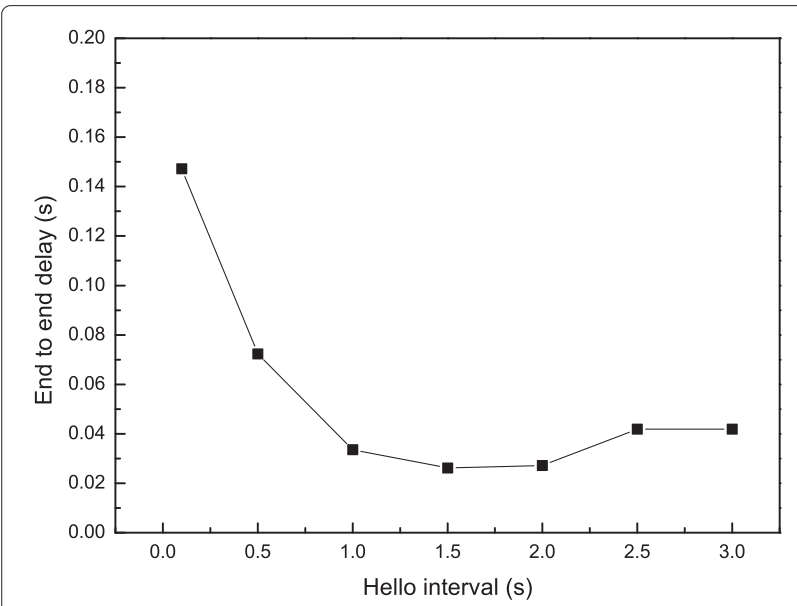

Figure 8 End-to-end delay vs. the Hello interval. 


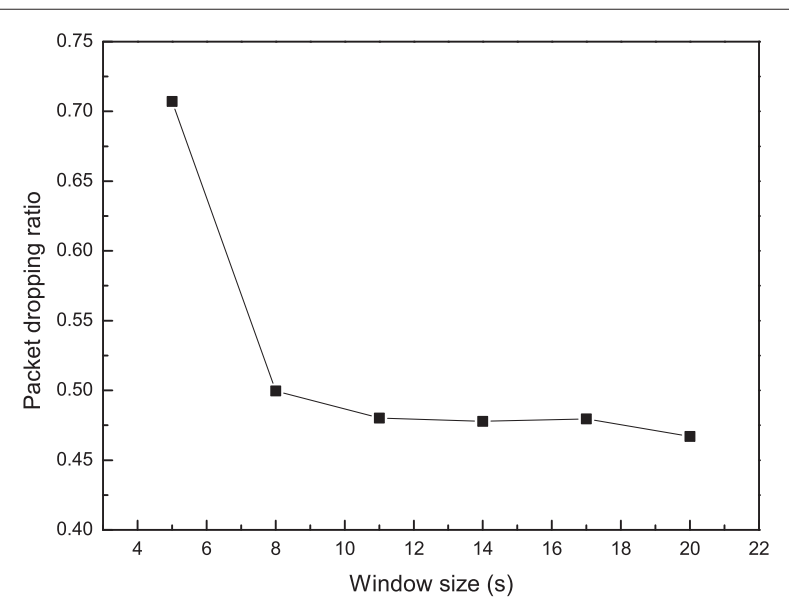

Figure 9 Packet dropping ratio vs. window size.

delay of LSGR. As shown in Figure 7, the packet dropping ratio reaches a high value when the Hello interval is short. It is because sending Hello packets frequently will consume too much resource and one vehicle can send one packet at the same time; other packets are prevented from being sent, thus the packet dropping rate turns out to be high. Then the packet dropping rate decreases with the increase of the Hello interval and reaches the lowest value at $1.5 \mathrm{~s}$. As shown in Figure 8, the end-to-end delay reaches a high value at first and then reduces with the increasing of the Hello interval, it is because the decrease of the queuing delay. Keeping on increasing the Hello interval causes higher packet dropping ratio and end-toend delay, because the longer the Hello interval is, the less accuracy of the measurement of the link state can be obtained, and with more packet dropping ratio, more time will be consumed by the retransmissions of the dropping packets.

\subsubsection{The impact of window size $w$}

To explore the impact of the window size, the Hello interval is set to $1 \mathrm{~s}$. As can be seen from the results in Figure 9, when the value of $w$ increases, the packet dropping rate is in decreasing trend. It is because considering the link quality in a longer period of time will contribute to the accuracy of the measurement of the link state. While after the value of $11 \mathrm{~s}$, the increasing of window size generates only a modest improvement in packet delivery rate, since the mobility of vehicles makes it difficult for connections between them to last a long period of time. The result shown in Figure 10 reveals that the changing trends of end-to-end delay and packet dropping ratio with window size are the same. These results contribute to choose better values of the Hello interval and window size to maximize the performance of LSGR.

\subsubsection{Hop count}

As shown in Figure 11, the hop count needed by LSGR is larger than that of GyTAR and GpsrJ+. In the case of low vehicle density, the hop count needed by LSGR is about one more than GyTAR. This is because LSGR will forward the packets through other road when a local optimum appears, whereas GyTAR just carry the packets until the next intersection or until another vehicle closer to the destination intersection enters in its transmission range. The transmission through other roads increases the hop count. However, since the radio travels much faster than vehicles, although LSGR takes more hop count, the

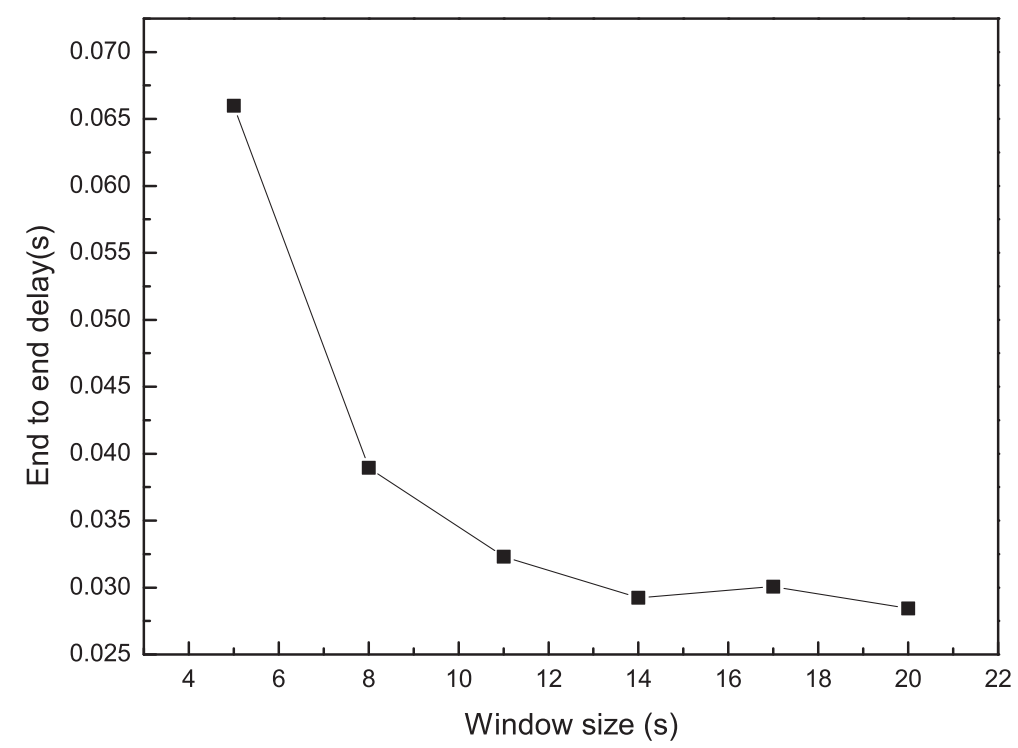

Figure 10 End-to-end delay vs. window size. 


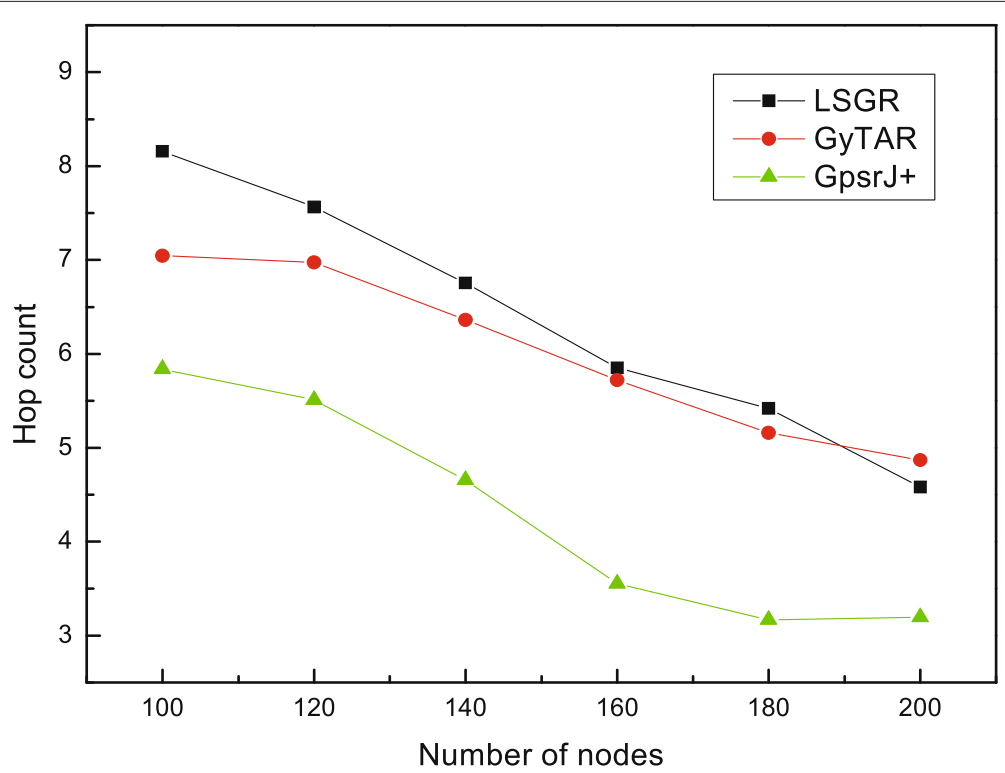

Figure 11 Hop count vs. the number of nodes.

end-to-end delay is lower than GyTAR (Figure 12). As the vehicle density becomes higher, the probability that a packet gets into a local optimum is reduced, the gap in hop count between LSGR and GyTAR is narrowed accordingly. The end-to-end delay saved by LSGR is mainly due to the reduced retransmission. The hop needed by LSGR is about two more than GpsrJ+. First, since LSGR chooses the next hop according to the EOA metric, the node that is located approximately to the transmission range of the forwarding node has less opportunity to be chosen as the next hop since it may have worsen the link quality. Therefore, more hops are needed for LSGR to send the packets to the destination. Secondly, by predicting on which road segment its neighboring junction node will forward packets to, packets in GpsrJ+ can bypass the junction without stopping on the junction node so as to reduce the hop count. Eventually, the hop count needed by GpsrJ+ is less than LSGR.

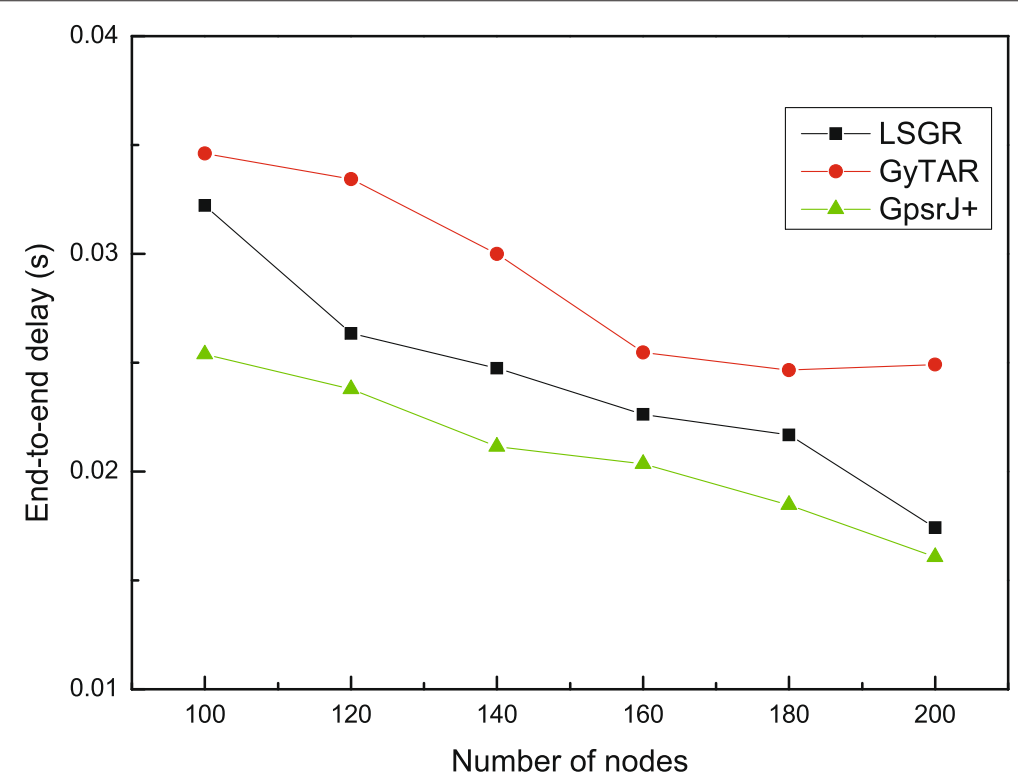

Figure 12 End-to-end delay vs. the number of nodes. 


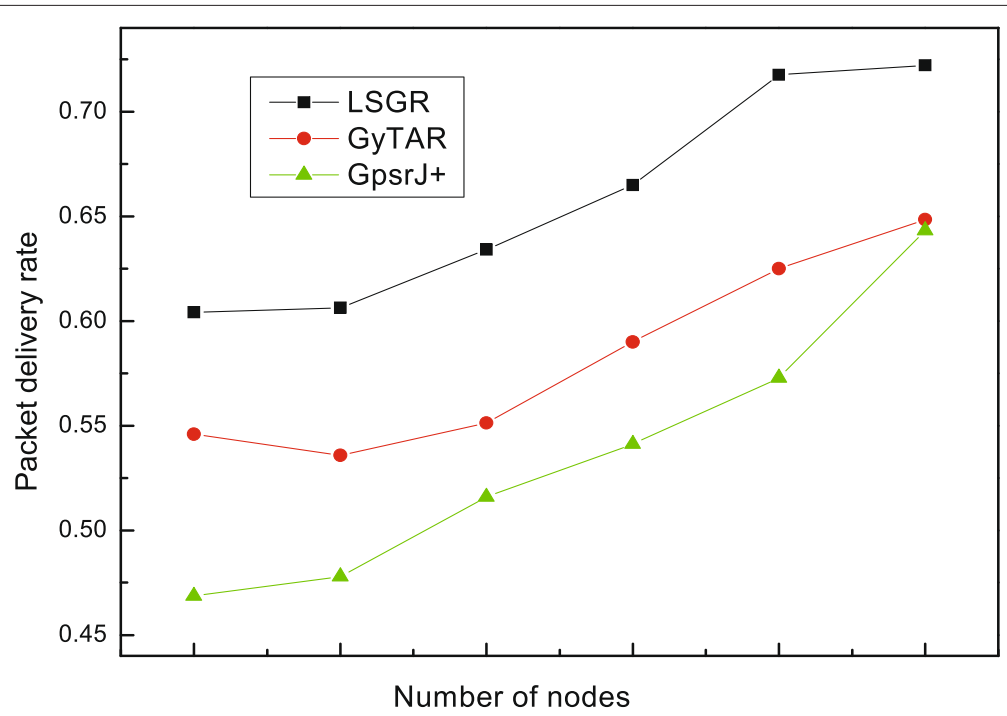

Figure 13 Packet delivery rate vs. the number of nodes.

\subsubsection{End-to-end delay}

As can be seen from the results shown in Figure 12, the delay achieved by LSGR is $20.9 \%$ lower than GyTAR on average. This superiority is due to the reason that in LSGR, the enhanced greedy forwarding takes the link quality into account when it chooses the next hop. Highquality links have greater chances to be chosen to forward the packets. The time needed to retransmit is saved, and accordingly, the end-to-end delay is shortened. In GyTAR, the improved greedy strategy predicts the position of each neighbor. According to the prediction, the neighbor clos- est to the destination intersection is selected as the next hop. However, the link between the neighbor and the forwarder may face a high packet dropping ratio due to the far distance between the two nodes. As a consequence, a considerable time would be wasted for packet retransmission. Moreover, in GyTAR, the recovery strategy is only based on the idea of 'carry and forward', whereas LSGR incorporates the idea of 'carry and forward' and the perimeter forwarding. Packets can exploit opportunities to recover from local optimum through other roads. The figure also shows that GpsrJ+ takes less time than GyTAR

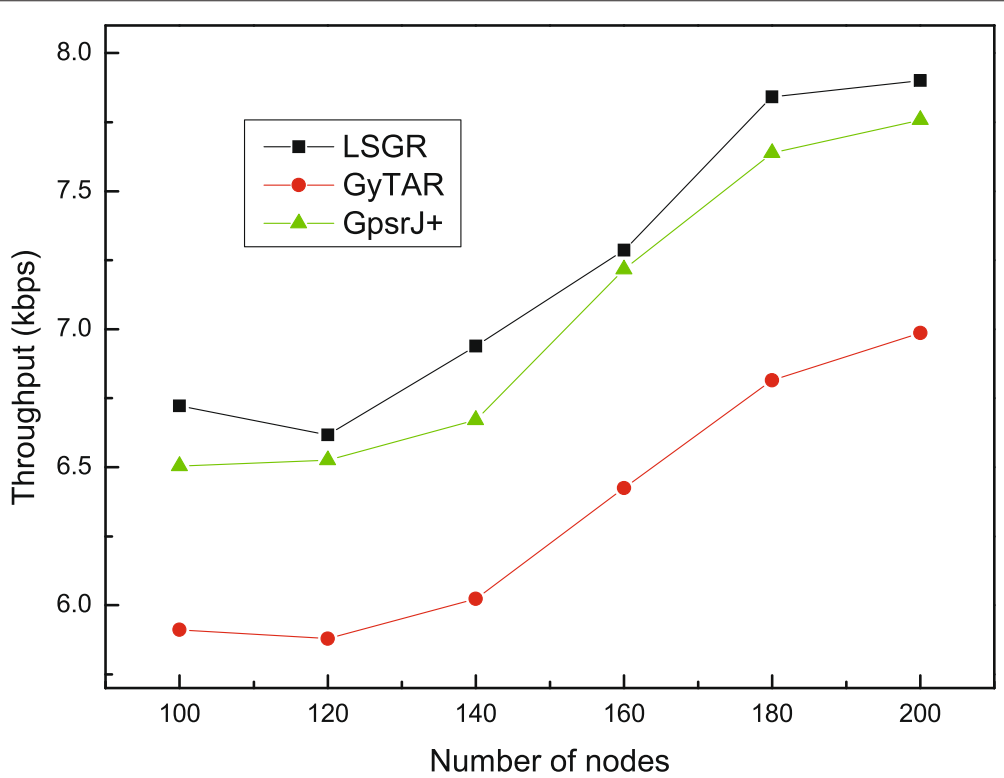

Figure 14 Network throughput vs. the number of nodes. 
and LSGR to transmit a packet from the source to the destination. The less hop count it needed contributes to the reduction.

\subsubsection{Packet delivery rate}

Figure 13 shows that LSGR achieves up to $9.17 \%$ and $22.66 \%$ improvement in packet delivery rate than GyTAR and GpsrJ+, respectively. The relative high-quality links used in LSGR contribute to the improvement. In LSGR, the next hop is selected by integrating the link's state and the geographic distance. Hence the nodes that have a bad-quality link with the forwarder have a smaller chance to be chosen as the next hop. GyTAR and GpsrJ+ choose the neighbor that is closest to the destination, the next hops obtained in such way are largely located close to the transmission range of the forwarder. Due to the channel fading, the links suffer a high packet dropping ratio. If the retransmission count in MAC layer reaches a threshold, the packet will be discarded. Consequently, the packet delivery rate achieved by GyTAR and GpsrJ+ is lower than LSGR. As can be seen from the figure, since LSGR and GyTAR consider the vehicle density to select the streets, the delivery rates of these two protocols are higher than those of GpsrJ+. As the number of nodes in the network increases, the chance that a packet encounters a local optimum is decreased, accordingly the packet dropping due to the lack of next hop is reduced. The comprehensive consideration on the choice of the next hop in LSGR contributes to the high packet delivery rate.

\subsubsection{Network throughput}

Figure 14 illustrates that LSGR improves the network throughput compared with GpsrJ+ and GyTAR. On average, LSGR can achieve 13.56\% higher than GyTAR and $2.35 \%$ higher than GpsrJ+. Taking the link quality into account when choosing the next hop reduces the retransmission count. The saved bandwidth can be utilized to transmit other packets, and consequently the network throughput is improved. However, as the next hop chosen by GyTAR and GpsrJ+ is confronted with high packet dropping from the forwarding node, an excessive amount of spectrum is consumed by retransmission. Other data packets cannot obtain the opportunity to be transmitted. As a result, the network throughput achieved is low.

\section{Conclusions}

In this paper, we have proposed a routing metric called EOA to enhance the greedy forwarding. The EOA metric incorporates the distance and the link quality to choose the next hop. It tends to maximize the packet advance through one-hop transmission, thereby reducing the bandwidth consumed by retransmission. Based on the EOA metric, a routing protocol called LSGR is propounded for VANETs. In LSGR, the forwarding node chooses the intermediate node with better quality link in straight road and chooses the road segment with higher connectivity in the intersections. Indeed, LSGR can be well adapted to the unstable link state in VANETs. To validate the performance of the protocol, we have compared LSGR with GpsrJ+ and GyTAR via NS-2. The simulation results have revealed that LSGR can achieve a better performance in terms of packet delivery rate and network throughput. Numerically, compared with GyTAR, LSGR can reduce the end-to-end delay by $17.53 \%$ and improve the throughput by $13.56 \%$. Compared with GpsrJ+, LSGR can achieve a much higher packet delivery rate and throughput at the spend of a little more end-to-end delay.

\section{Competing interests}

The authors declare that they have no competing interests.

\section{Acknowledgements}

This work was supported by the National Natural Science Foundation of China under Grant No. 61271176 and No. 61401334, the National Science and Technology Major Project under Grant No. 2013ZX03004007-003, the Fundamental Research Funds for the Central Universities, and the 111 Project (B08038)

Received: 29 November 2013 Accepted: 1 October 2014 Published: 25 October 2014

\section{References}

1. G Karagiannis, O Altintas, E Ekici, G Heijenk, B Jarupan, K Lin, T Weil, Vehicular networking: a survey, tutorial on requirements, architectures, challenges, standards and solutions. IEEE Commun. Surv. Tutorials. 13(4), 584-616 (2011)

2. V Devarapalli, R Wakikawa, A Petrescu, P Thubert, Network mobility (NEMO) basic support protocol. Technical report, RFC 3963, January (2005)

3. R Baldessari, A Festag, J Abeillé, in Proceedings of the 7th IEEE International Conference on ITS Telecommunications (ITST'07). NEMO meets VANET: a deployability analysis of network mobility in vehicular communication (Riviera, French, June 2007, pp. 1-6

4. SCéspedes, X Shen, C Lazo, IP mobility management for vehicular communication networks: challenges and solutions. IEEE Commun. Mag. 49(5), 187-194 (2011)

5. JH Lee, T Ernst, Lightweight NEtwork MObility within PMIPv6 for transportation systems. IEEE Syst. Journal. 5(3), 352-361 (2011)

6. JH Lee, T Ernst, N Chilamkurti, Performance analysis of PMIPv6-Based NEtwork MObility for intelligent transportation systems. IEEE Trans. Vehicular Technol. 61(1), 74-85 (2012)

7. G Yan, S Olariu, A probabilistic analysis of link duration in vehicular ad hoc networks. IEEE Trans. Intell. Transport. Syst. 12(4), 1227-1236 (2011)

8. SF Hasan, X Ding, NH Siddique, S Chakraborty, Measuring disruption in vehicular communications. IEEE Trans. Vehicular Technol. 60(1), 148-159 (2011)

9. R Oliveira, M Luis, L Bernardo, R Dinis, P Pinto, in Proceedings of the 2010 IEEE Wireless Communications and Networking Conference (WCNC). The impact of node's mobility on link-detection based on routing hello messages (Sydney, Australia, April, 2010), pp. 1-6

10. BN Karp, HT Kung, in Proceedings of the 6th ACM International Conference on Mobile Computing and Networking (MOBICOM'00). GPSR: Greedy 
perimeter stateless routing for wireless networks (Boston,

Massachussetts, USA, August, 2000), pp. 243-254

11. C Lochert, M Mauve, H Fusler, H Hartenstein, Geographic routing in city scenarios. ACM SIGMOBILE Mob. Comp. Comm. Rev. 9(1), 69-72 (2005)

12. M Jerbi, SM Senouci, Y Ghamri-Doudane, T Rasheed, Towards efficient geographic routing in urban vehicular networks. IEEE Trans. Vehicular Technol. 58(9), 5048-5059 (2009)

13. J Chang, Y Li, W Liao, I Chang, Intersection-based routing for urban vehicular communications with traffic-light considerations. IEEE Wireless Commun. 19(1), 82-88 (2012)

14. JW Lee, CC Lo, SP Tang, MF Horng, YH Kuo, in Proceedings of the IEEE International Conference on Advanced Communication Technology (ICACT). A hybrid traffic geographic routing with cooperative traffic information collection scheme in VANET (Phoenix Park, Korea, February, 2011), pp. 1496-1501

15. KC Lee, J Haerri, U Lee, M Gerla, in IEEE Globecom Workshops. Enhanced perimeter routing for geographic forwarding protocols in urban vehicular scenarios (Washington, DC, USA, November, 2007), pp. 1-10

16. KC Lee, U Lee, M Gerla, Geo-opportunistic routing for vehicular networks. IEEE Commun. Mag. 48(5), 164-170 (2010)

17. G Fenu, M Nitti, Strategies to carry, forward packets in VANET. Commun. Comput. Info. Science. 166(1), 662-674 (2011)

18. C Zhao, C Li, L Zhu, H Lin, J Li, in Processing of the IEEE International Conference on Wireless Communication and Signal Processing (WCSP). A vehicle density, load aware routing protocol for VANETs in city scenarios (Huang Shan, China, October, 2012), pp. 1-6

19. M Zuniga, B Krishnamachari, in Proceedings of the IEEE Communications Society Conference on Sensor and Ad Hoc Communications and Networks. Analyzing the transitional region in low power wireless links (Santa Clara, California, USA, October, 2004), pp. 517-526

20. L Zhao, $\mathrm{HZhu}, \mathrm{YXu}, \mathrm{X} \mathrm{Li}$, in Proceedings of the IEEE International Conference on Intelligent Sensors, Sensor Networks and Information Processing. LEGR: a load-balanced, energy-efficient geographic routing for lossy wireless sensor network (Melbourne, Austrilia, April, 2004), pp. 119-124

21. H Chen, D Qian, W Wu, W Fang, in Proceedings of the IEEE International Conference on Computational Science and Engineering (CSE). Link-aware geographic routing in wireless sensor networks (Vancouver, Canada, August, 2009), pp. 937-942

22. DSJ De Couto, D Aguayo, J Bicket, R Morris, in Proceedings of the 9th ACM International Conference on Mobile Computing, Networking (MobiCom'03). A high throughput path metric for multi-hop wireless routing (San Diego, CA, USA, September, 2003), pp. 134-146

23. R Draves, J Padhye, B Zill, Comparison of routing metrics for static multi-hop wireless networks. ACM SIGCOMM Comput. Comm. Rev. 34(4), 133-144 (2004)

24. S Biswas, R Morris, ExOR: Opportunistic multi-hop routing for wireless networks. ACM SIGCOMM Comput. Comm. Rev. 35(4), 133-144 (2005)

25. E Rozner, J Seshadri, Y Mehta, L Qiu, SOAR: simple oortunistic adaptive routing protocol for wireless mesh networks. IEEE Trans. Mobile Computing. 8(12), 1622-1635 (2009)

26. M Al-Rabayah, R Malaney, A new scalable hybrid routing protocol for VANETS. IEEE Trans. Vehicular Technol. 61(6), 2625-2635 (2012)

27. Y Lin, Y Chen, $S$ Lee, Routing protocols in vehicular ad hoc networks: a survey and future perspectives. J. Info. Sci. Eng. 26(3), 913-932 (2010)

28. F Li, Y Wang, Routing in vehicular ad hoc networks: a survey. IEEE Vehicular Technol. Mag. 2(2), 12-22 (2007)

29. MK Marina, SR Das, in Proceedings of the 9th IEEE International Conference on Network Protocols. On-demand multipath distance vector routing in ad hoc networks (California, USA, November, 2001), pp. 14-23

30. J Toutouh, J Garca-Nieto, E Alba, Intelligent OLSR routing protocol optimization for VANETs. IEEE Trans. Vehicular Technol. 61(4), 1884-1894 (2012)

31. H Saleet, O Basir, R Langar, R Boutaba, Region-based location-service-management protocol for VANETs. IEEE Trans. Vehicular Technol. 59(2), 917-931 (2010)

32. GG Finn, Routing and addressing problems in large metropolitan-scale internetworks, No. ISI/RR-87-180, University of Southern California Marina Del Rey Information Sciences Institute, Technical report (1987)
33. J Zhao, G Cao, VADD: Vehicle-assisted data delivery in vehicular ad hoc networks. IEEE Trans. Vehicular Technol. 57(3), 1910-1922 (2008)

34. The Network Simulator - ns-2. http://www.isi.edu/nsnam/ns/

35. J Haerri, F Filali, C Bonnet, M Fiore, in Proceedings of the ACM 3rd International Workshop on Vehicular ad hoc Network (VANET'06). VanetMobiSim: generating realistic mobility patterns for VANETs (New York, USA, September, 2006), pp. 96-97

doi:10.1186/1687-1499-2014-176

Cite this article as: Li et al:: A link state aware geographic routing protocol for vehicular ad hoc networks. EURASIP Journal on Wireless Communications and Networking 2014 2014:176.

\section{Submit your manuscript to a SpringerOpen ${ }^{\circ}$ journal and benefit from:}

- Convenient online submission

Rigorous peer review

- Immediate publication on acceptance

- Open access: articles freely available online

- High visibility within the field

- Retaining the copyright to your article

Submit your next manuscript at $\boldsymbol{\nabla}$ springeropen.com 